\title{
AVALIAÇÃO DA EQUIVALÊNCIA FARMACÊUTICA DE COMPRIMIDOS DE NIMESULIDA
}

\section{PHARMACEUTICAL EQUIVALENCE EVALUATION IN NIMESULIDA TABLETS}

\author{
Dianeffer KUROVSKI누, Vinícius Bednarczuk de OLIVEIRA²
}

\begin{abstract}
1 - Acadêmica do curso de Farmácia, Centro Universitário Campos de Andrade, Pós graduanda em Saúde Estética e Cosmética, Instituto Brasil de Pós-Graduação, Curitiba, Paraná, Brasil.

2 - Professor do Centro Universitário Campos de Andrade, Doutor em Ciências Farmacêuticas - UFPR, Curitiba, Paraná, Brasil.

Autor para correspondência: dieny_k@hotmail.com
\end{abstract}

\section{RESUMO:}

No mercado farmacêutico existe uma grande variedade de fármacos destinados para uma mesma patologia, havendo muitas vezes preocupação dos profissionais de saúde que os prescrevem, e também dúvida dos pacientes quanto a eficácia terapêutica de um medicamento genérico e similar a um de referência. A nimesulida é um fármaco que está disponível no mercado em várias formas farmacêuticas, é mais utilizada em forma sólida, como comprimidos de administração oral. O objetivo deste trabalho foi realizar testes de equivalência farmacêutica utilizando ensaio farmacopéicos. Os ensaios realizados foram de peso médio, dureza, friabilidade e doseamento por titulação. Os resultados demonstraram uma conformidade entre as amostras analisadas, sendo a friabilidade o resultado que mais apresentou diferenças, porém dentro do aceito pela farmacopeia Brasileira. Estes resultados são importantes demonstrando qualidade das amostras analisadas.

Palavras-chave: Equivalência farmacêutica; nimesulida; controle de qualidade.

\begin{abstract}
:
In the pharmaceutical market there is a great variety of drugs destined to the same pathology, there is often concern of the health professionals who prescribe them, as well as the doubts of the patients as to the therapeutic efficacy of a generic and similar drug to a reference. Nimesulide is a drug which is commercially available in various pharmaceutical forms, it is most commonly used in solid form, as tablets of oral administration. The objective of this work was to perform pharmaceutical equivalence tests using pharmacopoeial assays. The tests were of medium weight, hardness, friability and titration. The results demonstrated a conformity between the samples analyzed, being the friability the result that presented the most differences, however within the accepted by the Brazilian pharmacopoeia. These results are important demonstrating the quality of the analyzed samples.
\end{abstract}

Keywords: Pharmaceutical equivalence; nimesulida; quality control.

\section{INTRODUÇÃO}

A qualidade de um fármaco é de caráter comercial, legal e moral, o não cumprimento dos requisitos de qualidades podem acarretar sérias implicações, como falta de eficácia no tratamento ou efeitos tóxicos (KÖHLER et al, 2009). De acordo com a Lei 
9.787 de 10 de fevereiro de 1999, os medicamentos industrializados no Brasil passaram a serem classificados como medicamentos de referência, genérico e similar, a lei também estabeleceu a comercialização de medicamentos genéricos, sendo um análogo e intercambiável ao de referência (BRASIL, 1999). AANVISA adota em 02 de março de 2007 a RDC $n^{\circ} 17$, determinando que além dos medicamentos genéricos, os similares também deveriam ser submetidos a testes de bioequivalência, e os resultados comparados ao medicamento de referência (BRASIL, 2007).

A legislação brasileira exige que os medicamentos similares e genéricos sejam bioequivalentes ao seu medicamento de referência. Uma das etapas necessárias para esta comprovação são os estudos de equivalência farmacêutica, que se constituem em ensaios de controle de qualidade in vitro comparativos entre o medicamento teste e seu medicamento de referência (PUGENS et al, 2008).

O medicamento referência sendo o produto inovador, devendo ter sua qualidade e segurança aprovada cientificamente no momento do registro, junto ao órgão federal competente, podendo então ser comercializado e utilizado em testes comparativos de equivalência farmacêutica e/ou bioequivalência para a permissão de registros de medicamentos genéricos e similares (BRASIL, 1999; BRASIL, 2004).

Medicamento similar é aquele que apresenta paridade ao medicamento referência, mesmo princípio ativo, concentração, forma farmacêutica, via de administração, posologia e indicação terapêutica, podendo diferenciar-se somente quanto à forma e tamanho do produto, embalagem, rótulo, prazo de validade, excipientes e veículos, e comercializado sob nome comercial escolhido pelo fabricante (BRASIL, 1999).

Em 2014 por meio da RDC n58 ficou determinada a intercambialidade dos medicamentos similares com o medicamento de referência. Todos os medicamentos similares constantes na lista da ANVISA, passaram a ter na bula a informação a respeito da intercambialidade (BRASIL, 2014).

Existem contestações entre medicamentos de referência, genérico e similar, quanto ao emprego de matérias-primas, técnicas, processos e equipamentos, os quais ficam a critério do laboratório produtor, porém, essas diferenças não devem comprometer a bioequivalência do medicamento (KLEINKAUF, 2006; BRASIL, 2007).

Devido a grande competição, o mercado farmacêutico busca diante dos fabricantes, assegurar aos consumidores a qualidade e confiabilidade dos medicamentos produzidos, pois similares e genéricos ainda são alvos de questionamentos (LIMA, CAVALCANTI, 2009). 
A nimesulida é amplamente utilizada no Brasil, o que torna relevante avaliar a sua qualidade em diversas formulações, visando garantir a eficácia e segurança. A avaliação da qualidade de comprimidos representa uma etapa imprescindível para que haja a liberação para comercialização do medicamento em condições que garantam a segurança, eficácia terapêutica e a qualidade do produto, durante todo o prazo de validade (PEIXOTO et al, 2005). O fármaco nimesulida é um anti-inflamatório não esteroide (AINE), uma das classes de medicamentos mais prescritos em todo o mundo para tratamento de dor e inflamações (CARVALHO, 2010).

Diante deste contexto, o objetivo do presente artigo foi realizar estudo de qualidade farmacotécnica avaliando formas farmacêuticas orais sólidas da nimesulida a partir de ensaios físico-químicos.

\section{MATERIAL E MÉTODOS}

A realização do presente estudo foi no laboratório do Centro Universitário Campos de Andrade. As amostras foram analisadas por triplicata, utilizando cinco laboratórios distintos de nimesulida 100 mg de liberação imediata. Sendo dois laboratórios similares, dois laboratórios genéricos em comparativo com o medicamento referência disponível na Lista de Medicamentos da Anvisa.

As amostras foram analisadas empregando os ensaios exigidos pelos compêndios oficiais de peso médio, doseamento, dureza e friabilidade seguindo os limites estabelecidos na Farmacopéia Brasileira com algumas adaptações.

Os equipamentos utilizados foram: Balança Analítica Bioprecisa Modelo FA2104N; Friabilômetro Nova Ética Modelo 300; Durômetro Manual Nova Ética Modelo 0020819. Os reagentes utilizados foram os seguintes: Hidróxido de Sódio 0,1 $M$, Acetona, Fenolftaleína, Água.

A determinação de peso médio inicial foi feita pesando-se na Balança Analítica, individualmente três comprimidos de cada uma das amostras testadas de Nimesulida 100 mg (FARMACOPÉIA BRASILEIRA V, 2010).

Para o teste de friabilidade utilizou-se três comprimidos de cada amostra por vez, sendo classificados em: Referência, Similar 1, Similar 2, Genérico 1 e Genérico 2. Os comprimidos foram submetidos a ação do aparelho Friabilômetro por quatro minutos, o que corresponde a 100 rotações. Após todas as amostras passarem pela análise de friabilidade, foram pesadas novamente individualmente na Balança Analítica para determinação de 
peso médio final (MANFREDI; 2009, FARMACOPÉIA BRASILEIRA V, 2010).

O teste de dureza foi realizado submetendo-se três comprimidos de cada uma das amostras de Nimesulida 100 mg (Referência, Similar 1, Similar 2, Genérico 1, Genérico 2), individualmente à ação do aparelho Durômetro Manual, medindo a força necessária para esmagá-los, esse teste aplica-se principalmente a comprimidos não revestidos, permitindo determinar a sua resistência ao esmagamento ou a ruptura sob pressão radial (FARMACOPÉIA BRASILEIRA V, 2010).

O ensaio de doseamento das amostras de nimesulida foi conduzido de acordo com o método A (titulação) descrito na Farmacopéia Brasileira, V edição, volume II (2010). O comprimido foi triturado individualmente e adicionado a um erlenmeyer, juntamente com 30 $\mathrm{mL}$ de acetona previamente neutralizada, $20 \mathrm{~mL}$ de água e cerca de 3 gotas de fenolftaleína como indicador. Com uma pipeta graduada titulou-se com hidróxido de sódio a amostra até atingir coloração rosa.

\section{RESULTADOS E DISCUSSÃO}

Os resultados dos ensaios físico-químicos realizados encontram-se na tabela 1.

TABELA 1 - Resultados obtidos nas amostras analisadas.

\begin{tabular}{l|cccc}
\hline Amostra & $\begin{array}{c}\text { Peso Médio } \\
(\mathbf{m g} \pm \mathbf{D P})\end{array}$ & $\begin{array}{c}\text { Friabilidade } \\
(\% \pm \mathbf{D P})\end{array}$ & $\begin{array}{c}\text { Dureza } \\
\mathbf{( K g} \pm \mathbf{D P})\end{array}$ & $\begin{array}{c}\text { Doseamento } \\
\mathbf{( m g / c p} \pm \mathbf{D P})\end{array}$ \\
\hline Referência & $0,40 \pm 0,0005$ & $0,45 \pm 0,17$ & $6,50 \pm 0,50$ & $110,99 \pm 3,08$ \\
Similar 1 & $0,40 \pm 0,0006$ & $0,77 \pm 0,11$ & $6,53 \pm 0,50$ & $110,99 \pm 0,02$ \\
Similar 2 & $0,40 \pm 0,0047$ & $0,40 \pm 0,14$ & $5,33 \pm 0,31$ & $117,16 \pm 0,03$ \\
Genérico1 & $0,40 \pm 0,0016$ & $0,20 \pm 0,03$ & $6,97 \pm 0,50$ & $110,99 \pm 6,17$ \\
Genérico2 & $0,40 \pm 0,0026$ & $0,15 \pm 0,10$ & $6,17 \pm 0,46$ & $117,16 \pm 3,09$ \\
\hline
\end{tabular}

Segundo a Farmacopéia Brasileira V volume I (2010), o peso médio dos comprimidos pode ter uma variação de no máximo $7,5 \%$ para que sejam aprovados, os comprimidos de ambas as amostras analisadas: Referência, Similar 1, Similar 2, Genérico 1 e Genérico 2, apresentaram valor de peso semelhante, sendo de $400 \mathrm{mg}$ por comprimido.

O teste de friabilidade permite avaliar a resistência dos comprimidos ao atrito, garantindo que se manterão íntegros durante os processos de acondicionamento, 
revestimento, emblistagem e transporte (MELO et al., 2006). São consideráveis aceitáveis os comprimidos com perda igual ou inferior a $1,5 \%$ do seu peso (FARMACOPÉIA BRASILEIRA V, 2010). Todas as amostras analisadas apresentaram valores dentro do especificado pelo compêndio referenciado, sendo o similar 1 o que mais perdeu massa neste ensaio e os genéricos os medicamentos que apresentaram os menores valores.

De acordo com Cazedey (2012), quanto maior for a dureza menor será a porosidade apresentada pelo comprimido, o que consequentemente, irá resultar na dificuldade do líquido de dissolução penetrar na matriz, e mais lenta será a liberação do fármaco para o meio. A literatura oficial não traz padrões máximos para este teste, indicando que o ensaio é apenas informativo. Entre as amostras analisadas o medicamento similar 2 foi o que apresentou o menor valor de dureza, apresentando 23,53\% menos dureza que o medicamento genérico 1.

Para Xavier e Sousa (2013), o doseamento de um fármaco é um dos ensaios mais importantes para se avaliar a qualidade dos produtos farmacêuticos, pois determina a quantidade de princípio ativo presente na formulação. Nas amostras analisadas esperavase uma quantidade de $100 \mathrm{mg}$ de princípio ativo em cada comprimido, nos ensaios de titulação o resultado variou entre 110,99 mg e 117,16 mg. A Farmacopéia Brasileira V Volume I (2010) estabelece uma variância mínima de 95,0 \% e máxima de 105,0 \% de teor do fármaco.

A conformidade dos medicamentos com as especificações farmacopéicas garante a qualidade, eficácia e segurança dos mesmos, e é um atributo de caráter não apenas comercial, mas também legal e moral. O controle de qualidade é a principal ferramenta para assegurar a intercambialidade entre os medicamentos, enquanto a qualidade para muitos produtos é uma questão de competitividade, no campo da saúde deve ser obrigatoriamente atendida, pois o não cumprimento de especificações de qualidade consideradas imprescindíveis pode acarretar sérias implicações tais como, falta da eficácia no tratamento e efeitos tóxicos (GIL, 2010).

\section{CONCLUSÃO}

Os resultados atingiram as expectativas, evidenciando qualidade por parte dos medicamentos analisados. Apesar de terem apresentado valor acima do preconizado pela literatura no perfil de doseamento, não é possível condenar sua eficácia terapêutica considerando apenas esse atributo de qualidade. O profissional farmacêutico tem 
importante papel nesse cenário de monitoramento da qualidade de medicamentos por meio de pesquisas científicas, e deve continuar desenvolvendo estudos que beneficiem o acesso a informação para a população, uma vez que é ele quem atua em contato direto com o paciente na orientação do uso de medicamentos, e presta os mais diversos serviços de assistência e cuidado farmacêutico.

\section{REFERÊNCIAS}

BRASIL. Ministério da Saúde. Agência Nacional de Vigilância Sanitária. Lei no 9.787 de 10 de fevereiro de 1999. Dispõe sobre a vigilância sanitária, estabelece o medicamento genérico, dispõe sobre a utilização de nomes genéricos em produtos farmacêuticos e dá outras providências. Diário Oficial da União. Brasília: Poder Executivo; 1999.

BRASIL. Ministério da Saúde. Agência Nacional de Vigilância Sanitária. Resolução RDC no 133 de 29 de maio de 2003. Regulamento técnico para medicamentos genéricos, 2003.

BRASIL. Ministério da Saúde. Agência Nacional de Vigilância Sanitária. Resolução da Diretoria Colegiada no 310, de 01 de setembro de 2004. Guia para realização do estudo e elaboração do relatório de equivalência farmacêutica e perfil de dissolução. Diário Oficial da União. Brasília: Poder Executivo; 2004.

BRASIL. Ministério da saúde. Agência Nacional de Vigilância Sanitária. Resolução da Diretoria Colegiada no 17, de 02 de março de 2007. Regulamento técnico para registro de medicamento similar. Diário Oficial da União. Brasília: Poder Executivo; 2007.

BRASIL. Ministério da Saúde. Agência Nacional de Vigilância Sanitária. Resolução da Diretoria Colegiada oㅡ 58, de 10 de outubro de 2014. Regulamento técnico para intercambialidade de medicamentos similares com o medicamento de referência. Diário Oficial da União. Brasília: Poder Executivo; 2014.

CARVALHO, W, A. Anti-inflamatórios não esteroides, analgésicos, antipiréticos e drogas utilizadas no tratamento da gota. In__ SILVA, P. Farmacologia. 8. ed. Rio de Janeiro: Guanabara Koogan, 2010. 
CAZEDEY, E. C. L. Análise químico-farmacêutica e estudos de estabilidade e de dissolução de comprimidos de orbifloxacino. 2012. 283 f. Tese de Doutorado (Programa de Pós-graduação em Ciências Farmacêuticas) - Universidade Estadual Paulista. "Júlio de Mesquita Filho", Araraquara, 2012.

FARMACOPÉIA BRASILEIRA; Agência Nacional de Vigilância Sanitária. Brasília: Anvisa, volume 1. 5ª Ed., p. 1159. 2010.

FARMACOPÉIA BRASILEIRA; Agência Nacional de Vigilância Sanitária. Brasília:

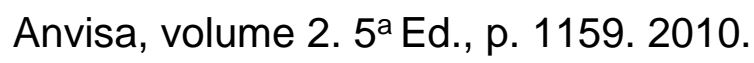

GIL, E. S. Controle físico-químico de qualidade de medicamentos. 3. ed. São Paulo: Pharmabooks, 2010.

KLEINKAUF RD. Desenvolvimento e validação de ensaios de dissolução para cápsulas de liberação prolongada de cloridrato de venlafaxina e de qualidade entre medicamento genérico e referência [Monografia]. Novo Hamburgo: Centro Universitário Feevale; 2006.

KÖHLER, L. F., et al. Avaliação biofarmacotécnica e perfil de dissolução de comprimidos de dipirona: equivalências farmacêuticas entre medicamentos de referência, genéricos e similares. Rev. Bras. Farm, v. 90, n. 4, p. 309-315. 2009.

LIMA J.P.R, C. F; P.F. Indústria farmacêutica: a evolução recente no Brasil e o caso de Pernambuco. Rev Bras Estud Urbanos Reg. 2009; 1(1).

MANFREDI, M. A. Estudo comparativo dos métodos de doseamento de comprimidos de ácido acetilsalicílico (AAS). 2009. 60 f. Trabalho de Conclusão de Curso (Graduação em Farmácia) - Universidade de Mogi das Cruzes, Mogi das Cruzes, 2009.

MELO, EB, et. al. Medicamentos similares e saúde pública: controle de qualidade físicoquímico de comprimidos de similar de ácido acetilsalicílico do estoque da farmácia básica do município de Cascavel, PR, Brasil. Acta Farm Bonaerense, 25(3):344-50, 2006. 
PEIXOTO, M.M; et al. Avaliação da qualidade de comprimidos de captopril dispensados em Feira de Santana-BA. Infarma, 40 (13-14): 69-73, 2005.

PUGENS AM; DONADUZZI CM, MELO EB. Controle de qualidade total e equivalência farmacêutica de três apresentações de captopril. Rev Eletr. de Farm 2008; 5(1): 32-45.

XAVIER, M. P.; SOUSA, S. F. Análise do teor de ácido acetilsalicílico 100mg em comprimidos comercializados no município de Gurupi -TO. Revista Amazônia, Gurupi - TO, v. 1, n. 3, pp. 35-42, 2013. 